\title{
Anti-Inflammatory Effect of Musa acuminata Stem
}

\author{
Maharani Laillyza Apriasari ${ }^{1}$ \\ Selviana Rizky Pramitha \\ ${ }^{1}$ Department of Oral Medicine, Faculty of Dentistry, \\ Lambung Mangkurat University, Kalimantan Selatan, Indonesia \\ 2Department of Dental Material, Faculty of Dentistry, \\ Lambung Mangkurat University, Kalimantan Selatan, Indonesia \\ ${ }^{3}$ Department of Oral Medicine, Faculty of Dental Medicine, \\ Universitas Airlangga, Surabaya, Indonesia
}

\author{
Dewi Puspitasari ${ }^{2}$ Diah Savitri Ernawati ${ }^{3}$
}

\begin{abstract}
Address for correspondence Diah Savitri Ernawati, drg., MSi. Sp.PM(K), Department of Oral Medicine, Faculty of Dental Medicine, Universitas Airlangga; Jl. Mayjend. Prof. Dr. Moestopo No.47, Surabaya 60132, Indonesia (e-mail: diah-s-e@fkg.unair.ac.id).
\end{abstract}

Eur J Dent 2020;14:294-298

\begin{abstract}
Keywords

- Musa acuminata

- anti-inflammation

- TNF- $\alpha$

$-\mathrm{NF}-\mathrm{KB}$

Objective This study was designed to assess the anti-inflammatory effect of Musa acuminata through the expression of tumor necrosis factor- $\alpha$ (TNF- $\alpha$ ) and nuclear factor kappa $\beta$ (NF-KB) after 3 days of application of Musa acuminata stem extract (MASE) gel on oral mucosal wound.

Materials and Methods An experimental study with post-test only control group design was conducted. Twenty male Rattus norvegicus (Wistar) were injured on their left buccal mucosa and treated three times a day with MASE gel of varying concentrations: $0 \%$ (as control), MASE 25\%, MASE 37.5\%, and MASE 50\%. On day 3, a biopsy was performed on each mucosal wound for later immunohistochemical analysis for the expressions of TNF- $\alpha$ and NF-KB.

Results The highest expression of TNF- $\alpha$ was observed in the control group $(13.20 \pm 1.79)$, while the lowest was in the treatment group using 50\% MASE $(6.40 \pm 1.14)$. Meanwhile the comparison between treatment groups did not highlight any significant difference $(p>0.05)$. The highest expression of NF-KB was observed in the control group (13.20 \pm 1.30$)$, whereas the lowest was in the treatment group using MASE 50\% (6.40 \pm 1.14$)$. NF-KB was significantly lower in the treatment group using MASE 50\% when compared with other treatment groups $(p<0.05)$.

Conclusion Application of MASE on mucosal wound reduces the expression of TNF- $\alpha$ and NF-KB at all concentrations. The anti-inflammatory effect of MASE $50 \%$ was the strongest one.
\end{abstract}

\section{Introduction}

Indonesia is known as a country that has a wide diversity of biological species. The country also has one of the richest store houses of medicinal plants in the world, despite research and development of these plants remaining extremely limited. Drugs derived from plants, also known as herbal medicines, can be used as alternatives to chemical drugs due to their lower potential for inducing adverse effects. Moreover, many plants and their extracts are also used for wound treatment. ${ }^{1}$

Banana (Musa spp.) is one of the most popular fruits in industrialized counties. Musa spp. is widely used as a cooking ingredient, especially in desserts. One type of Musa widely cultivated in South Borneo, Indonesia, is Mauli banana (Musa acuminata), which is very popular due to its sweet and appetizing taste. Interestingly, the stem of Musa acuminata contains many bioactive components such as ascorbic acid, $\beta$-carotene, lycopene, saponin, alkaloid, flavonoid, and tannin. ${ }^{2}$

Previous studies on Musa acuminata revealed that its stem extract (MASE) has antibacterial, antifungal, and antioxidant properties. $^{3}$ MASE of $25 \%$ concentration exhibited strong antibacterial and antifungal properties against Streptococcus mutants and Candida albicans. ${ }^{4}$ The antioxidant property was achieved through the binding of MASE's bioactive compounds with ferrous iron, hydrogen peroxide, and hydroxyl. 
In addition, MASE was also shown to significantly downregulate malondialdehyde and upregulate super oxide dismutase. ${ }^{3}$ A cytotoxicity test of MASE on baby hamster kidney-21 fibroblast cells showed nontoxic properties. ${ }^{2,3}$ Moreover, MASE gel of $37.5 \%$ concentration can induce epithelium and fibroblast proliferation, as well as vascular endothelial growth factor in mucosal wounds of Rattus norvegicus, thus accelerating the wound-healing process. ${ }^{4,5}$

The most abundant bioactive compound in MASE is tannin, which contains polyphenols. ${ }^{2,3}$ The polyphenol showed anti-inflammatory effects through the inhibitory mechanism of tumor necrosis factor $\alpha$ (TNF- $\alpha$ ) and nuclear factor kappa $\beta$ (NF-KB) signal. ${ }^{6}$ The TNF- $\alpha$ is a pro-inflammatory cytokine produced by activating the NF-KB.7 The inhibition of NF-KB and TNF- $\alpha$ is the key for the anti-inflammatory effect to increase the oral mucosal wound healing. ${ }^{8}$ This study was therefore designed to evaluate the anti-inflammatory effect of Musa acuminata through expression of TNF- $\alpha$ and NF-KB after 3 days of application of MASE on oral mucosal wounds.

\section{Materials and Methods}

This study was an experimental laboratory research incorporating post-test only control group design. Research procedures were approved by the Ethical Clearance Committee, Faculty of Dentistry, Universitas Lambung Mangkurat; Banjarmasin, South Borneo, Indonesia, with number 039/KEPKG-FKGULM/ EC/IX/2017.

\section{Preparation of MASE}

Extraction of Musa acuminata's stem (MASE) was performed in several steps. Stems of Musa acuminata were washed under running water, cut into small pieces, and dried in an oven at temperatures of 40 to $60^{\circ} \mathrm{C}$ for 3 days. The dried stems were then smoothed in a blender and soaked in $70 \%$ ethanol for 72 hours (maceration method) to produce the extract. The extract was agitated and filtered every day before being evaporated twice in a vacuum rotatory evaporator at temperatures of 40 to $50^{\circ} \mathrm{C}$ and a water bath until a thick extract was obtained. The resulting ethanol-free extract was then weighed and used to produce $0 \%, 25 \%, 37.5 \%$, and $50 \%$ gel concentrations by adding $15 \%$ hydroxypropyl cellulose medium, $1 \%$ tween $80,8 \%$ propylene glycol, 5 drops of candy oil, and distilled water until 100\% weight was reached.

\section{In Vivo Study}

Twenty male Rattus norvegicus (Wistar) rats were randomly divided into four groups. Their left buccal mucosae were injured by means of a punch biopsy with a $6 \mathrm{~mm}$ diameter and $1 \mathrm{~mm}$ depth after being placed under general anesthesia ether. ${ }^{5}$ The wound was ensured to be confined to the epithelium, without damaging the underlying muscle. Each of the four groups was then treated with MASE gel of varying concentrations three times a day (every 6-8 h); 0\% (as control), MASE 25\%, MASE 37.5\%, and MASE 50\%.

After 3 days of application, the Rattus norvegicus were sacrificed with lethal dose of ether by inhalation and their buccal mucosae biopsied for immunohistochemistry (IHC) analysis. Immunohistochemical staining was conducted using anti-mouse TNF- $\alpha$ monoclonal antibodies (Santa Cruz Biotechnology Inc, TNF- $\alpha$ [M-18]: sc 1348) and NF-KB monoclonal antibodies (Santa Cruz Biotechnology Inc, NF-KB p65 [F-6]: sc 8008). The number of macrophage cells showing the expressions of TNF- $\alpha$ and NF-KB was calculated in three different field locations using a light microscope (Olympus, United States) at 400× magnification, and subsequently averaged.

\section{Statistical Analysis}

The results were analyzed using one-way analysis of variance parametric test based on Shapiro-Wilk normality test and Levene's variance homogeneity test. The results showed normal data distribution and homogenous data variances. Consequently, further analysis by means of a Post Hoc Least Significant Difference test was conducted with significance $p<0.05$.

\section{Result}

The anti-inflammatory effect of MASE on mucosal wounds was analyzed using IHC. The results of IHC analysis are shown in -Figs. 1 and 2.

The highest expression of TNF- $\alpha$ was observed in the control group (13.20 \pm 1.79 ), while the lowest was in the treatment group using MASE 50\% (6.40 \pm 1.14$)$. The highest expression of NF-KB was observed in the control group $(13.20 \pm 1.30)$, whereas the lowest was in the treatment group using MASE 50\% (6.40 \pm 1.14 ; - Table 1).

The expression of TNF- $\alpha$ in control group is higher compared to MASE $25 \%(p=0.000)$, MSE $37.5 \%(p=0.000)$, and MASE $50 \%(p=0.000)$. The expression of TNF- $\alpha$ comparison between treatment groups did not show any difference $(p>0.05$; - Table 1$)$.

The expression of NF- $\mathrm{KB}$ in control group is higher compared to MASE $25 \%(p=0.000)$, MSE $37.5 \%(p=0.000)$, and MASE 50\% $(p=0.000)$. The expression of NF-кB was lower in MASE 50\% compared to MASE 37.5\% $(p=0.045)$ and MASE 25\% ( $p=0.019 ;$ - Table 1$)$.

\section{Discussion}

Inflammation is a protective response to tissue injury and infection characterized by a series of host responses including: vasodilation, and recruitment of immune cells and plasma proteins to the injured tissue. ${ }^{9}$ Immune cells play a vital role in wound healing and contribute to the release of lysosomal enzymes and reactive oxygen species. In addition, these cells also clean the damaged area of debris. ${ }^{10}$

Cellular response induces pro-inflammatory mediators produced either from endogenous leukocytes (macrophages, monocytes, dendritic cells, or lymphocytes) and/or from the tissue cells themselves. These mediators are the main keys for inflammatory response, which is initiated by the recruitment of neutrophils. ${ }^{11}$ Neutrophils are the main immune cells in 

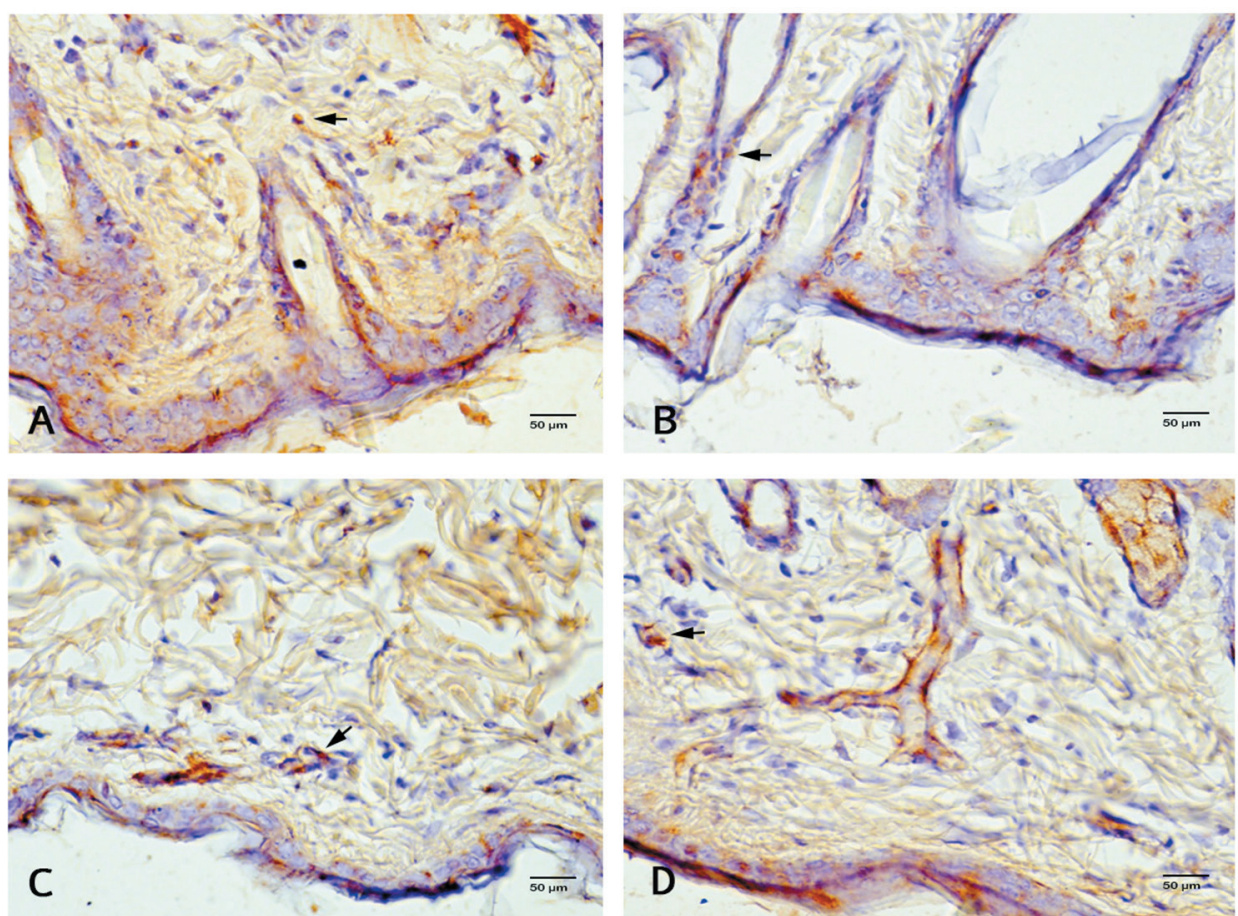

Fig. 1 Macrophage's expression of tumor necrosis factor- $\alpha$ (TNF- $\alpha$; black arrow) on control group (A), Musa acuminata stem extract (MASE) concentrations of $25 \%$ (B), MASE concentrations of $37.5 \%$ (C), and MASE concentrations of $50 \%$ (D).
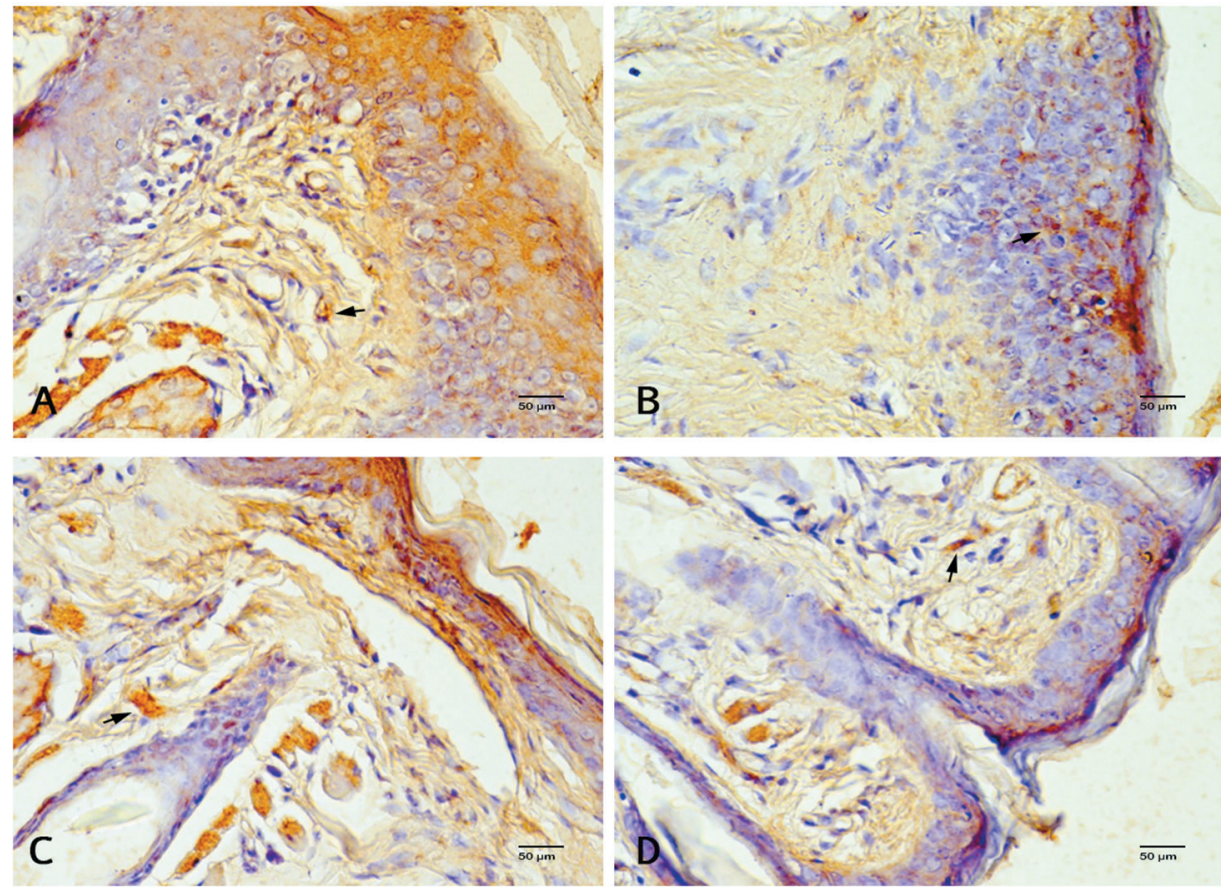

Fig. 2 Macrophage expression of nuclear factor kappa $\beta$ (NF-KB; black arrow) in control group (A), Musa acuminata stem extract (MASE) concentrations of $25 \%$ (B), MASE concentrations of $37.5 \%$ (C), and MASE concentrations of $50 \%$ (D).

the first inflammation phase ( $48 \mathrm{~h}$ after injury) and begin to wane after 24 to 36 hours due to apoptosis at the time the circulating monocytes enter the wound and mature into tissue macrophages that play a crucial role in wound healing. ${ }^{2}$ Macrophages are capable of inducing apoptosis of neutrophils with the help of membrane-bound NF- $\alpha, \beta 3$-integrins, and CD $36 .{ }^{13}$
Following neutrophils, monocytes will migrate to the wound site and become macrophage, which play a central role in both the inflammatory phase and all stages of repair. ${ }^{12}$ These cells are also responsible for regulating cellular components of the wound by inducing apoptosis or phagocytosing certain cells..$^{13}$ Meanwhile, macrophages phagocytose debris and bacteria, and produce and orchestrate inflammatory 
Table 1 The expression of TNF- $\alpha$ and NF-KB following 3 days of MASE application

\begin{tabular}{|l|l|l|}
\hline \multirow{2}{*}{ Group } & \multicolumn{2}{|c|}{ Mean \pm SD } \\
\cline { 2 - 3 } & Expression of TNF- $\alpha$ & Expression of NF-KB \\
\hline Control & $13.20 \pm 1.79^{\mathrm{a}, \mathrm{b}, \mathrm{c}}$ & $13.20 \pm 1.30^{\mathrm{a}, \mathrm{b}, \mathrm{c}}$ \\
\hline MASE 25\% & $6.60 \pm 1.81^{\mathrm{a}}$ & $9.00 \pm 1.22^{\mathrm{a}, \mathrm{d}}$ \\
\hline MASE 37.5\% & $7.20 \pm 0.83^{\mathrm{b}}$ & $8.60 \pm 1.51^{\mathrm{b}, \mathrm{e}}$ \\
\hline MASE 50\% & $6.40 \pm 1.14^{\mathrm{c}}$ & $6.40 \pm 1.14^{\mathrm{c}, \mathrm{d}, \mathrm{e}}$ \\
\hline
\end{tabular}

Abbreviations: MASE, Musa acuminata stem extract; NF-KB, nuclear factor kappa $\beta$; SD, standard deviation; TNF- $\alpha$, tumor necrosis factor- $\alpha$. Note: The same superscript character in each variable shows the differences for each group $(p<0.05)$.

$\mathrm{a}, \mathrm{b}, \mathrm{c}$ and $\mathrm{d}$ in TNF- $\alpha$ expression has value $p=0.000$

$a, b, c$ in NFkB expression has value $p=0.000$

$\mathrm{d}$ in NF-KB expression has value $p=0.019$

e in NFkB expression has value $p=0.045$

cytokines (including growth factors) such as TNF- $\alpha$, interleukin (IL)-6, IL-1, and basic fibroblast growth factor. ${ }^{12}$

TNF- $\alpha$, also known as cachectin, is an inflammatory cytokine that plays a key role in the inflammatory response. TNF acts on several different signaling pathways through two cell surface receptors, TNFR1 and TNFR2, to regulate apoptotic pathways, NF-KB activation of inflammation, and activate stress-activated protein kinases. ${ }^{14}$ Moreover, TNF- $\alpha$ represent the archetypal pro-inflammatory cytokines that are rapidly released following tissue injury or infection. TNF $\alpha$ leads the activation of RelA- or cRel-containing complexes in NF-KB. ${ }^{15}$

In this study, the anti-inflammatory effect of MASE gel on mucosal wounds through TNF- $\alpha$ and NF-KB expressions is evaluated. A study by Ritsu et $\mathrm{al}^{16}$ using a mouse model had previously shown that TNF- $\alpha$ production was detected at a peak concentration on day 3 after the inflicting of the wound. In keeping with this finding, the biopsy and IHC analysis contained in this study were also performed within the same time frame. ${ }^{16}$ The results of the study showed that the expression of TNF- $\alpha$ decreased with MASE-dose-dependent application. The MASE 50\% showed the lowest expression of TNF- $\alpha$.

TNF- $\alpha$ represents the pro-inflammatory cytokines that are immediately released after injury to tissues. The NF-KB pathway has been considered as the prototypical pro-inflammatory signaling pathway largely based on the activation of NF-KB by pro-inflammatory cytokines such as TNF- $\alpha{ }^{7}$ TNF- $\alpha$ can induce the degradation of NF-KB inhibitor (IKB) via a specific cascade pathway. ІкB sequesters NF-KB in the cytoplasmic compartment by binding to its inhibitory subunit. The breakdown of IкB therefore promotes the release of NF-KB that can translocate into the nucleus and activate specific inflammatory genes. Inflammation is a complex physiological process and the role of NF-KB in the inflammatory response cannot be extrapolated from in vitro studies. ${ }^{15-17}$

In the onset of inflammation, NF-KB promotes pro-inflammatory gene induction and also anti-inflammatory genes. In the nucleus, NF-кB binds to specific DNA targets to form what are known as $\mathrm{k} \beta$ sites. They are present in gene target, assimilate with the basal transcriptional machinery, and associate with other transcription factors, including AP-1 and chromatin remodeling proteins such as CREB binding protein and p300. An active NF-KB nuclear translocation can execute the role of a pro- and anti-inflammatory mediator. ${ }^{18}$ Prolonged inflammatory response can be resolved by the inhibition of NF-KB. ${ }^{16,19}$

This study showed that the expression of TNF- $\alpha$ decreased at all MASE concentrations compared with the control. Inhibition of TNF- $\alpha$ also decreased NF-кB expression in the macrophage cells involved in the mucosal wound-healing process. MASE gel contains polyphenol-possessed anti-inflammatory effect. The polyphenol can mediate the effect of TNF- $\alpha$ expression in macrophage cells by inhibiting p300/CREB-specific acetyl transferase and lead to the repression of the acetylation of histone/nonhistone proteins and histone acetyl transferase-dependent chromatin transcription. It may subsequently down-modulate the activation of $\mathrm{NF}-\mathrm{KB}{ }^{12}$

MASE 50\% has anti-inflammatory effects stronger than MASE 25\% and MASE 37.5\%. The application of MASE gel three times a day every 6 to 8 hours for 3 days can prevent the overexpression of TNF- $\alpha$ and NF-кB. The application of MASE 50\% can accelerate wound healing.

\section{Conclusion}

It can be concluded that during the wound-healing process, the highest anti-inflammatory effect is obtained after the application of MASE $50 \%$ for 3 days on the oral mucosal wound.

\section{Conflict of Interest}

None declared.

\section{Acknowledgment}

The authors sincerely thank the Ministry of Research, Technology and Higher Education of the Republic of Indonesia for the support given during the study.

\section{References}

1 Maver T, Maver U, Stana Kleinschek K, Smrke DM, Kreft S. A review of herbal medicines in wound healing. Int J Dermatol 2015;54(7):740-751

2 Apriasari ML, Iskandar SE. Bioactive compound and antioxidant activity of methanol extract Mauli bananas (Musa sp) stem. Int J Biosci Biochem Bioinform 2014;4(2):110-115

3 Fitriati Noor W, Aprianti N, Rizky Saputra S, Laillyza Apriasari $\mathrm{M}$, Suhartono E. Oxidative stress on buccal mucosa wound in rats and rule of topical application of ethanolic extracts of Mauli banana (Musa acuminata) stem. J Trop Life Sci 2015;5(2):84-87

4 Apriasari ML, Endariantari A, Oktaviyanti IK. The effect of $25 \%$ Mauli banana stem extract gel to increase the epithel thickness of wound healing process in oral mucosa. Dent J 2015;48(3):151-154 
5 Apriasari ML, Dachlan YP, Ernawati DS. Effect of musa acuminata stem by immunohistochemistry test in ulcer. Asian J Biochem 2016;11(3):135-141

6 Aggarwal BB, Gupta SC, Sung B. Curcumin: an orally bioavailable blocker of TNF and other pro-inflammatory biomarkers. Br J Pharmacol 2013;169(8):1672-1692

7 Surboyo MDC, Arundina I, Rahayu RP, Mansur D, Bramantoro T. Potential of distilled liquid smoke derived from coconut (Cocos nucifera L) shell for traumatic ulcer healing in diabetic rats. Eur J Dent 2019;13(2):271-279

8 Surboyo MDC, Ernawati DS, Arundina I, Rahayu RP. Oral ulcer healing after treatment with distilled liquid smoke of coconut shell on diabetic rats. J Krishna Inst Med Sci Univ 2019;8(2):70-79

9 Liu T, Zhang L, Joo D, Sun SC. NF-кB signaling in inflammation. Signal Transduct Target Ther 2017;2 doi: 10.1038/sigtrans. 2017.23

10 Gonzalez ACDO, Costa TF, Andrade ZA, Medrado ARP. Wound healing - a literature review. An Bras Dermatol 2016;91(5):614-620

11 Janssen WJ, Henson PM. Cellular regulation of the inflammatory response. Toxicol Pathol 2012;40(2):166-173

12 Qing C, The molecular biology in wound healing \& non-healing wound [Internet]. Vol. 20, Chinese Journal of Traumatology English Edition. Daping Hospital and the Research Institute of Surgery of the Third Military Medical University; 2017 p. 189-93. Available at: http://dx.doi.org/10.1016/j.cjtee. 2017.06.001

13 Brancato SK, Albina JE. Wound macrophages as key regulators of repair: origin, phenotype, and function. Am J Pathol 2011;178(1):19-25 [Internet]

14 Zhang JM, An J. Cytokines, inflammation, and pain. Int Anesthesiol Clin 2007;45(2):27-37

15 Lawrence T. The nuclear factor NF-kappaB pathway in inflammation. Cold Spring Harb Perspect Biol 2009;1(6):a001651

16 Ritsu M, Kawakami K, Kanno E, et al. Critical role of tumor necrosis factor- $\alpha$ in the early process of wound healing in skin. J Dermatology Dermatologic Surg [Internet] 2017;21(1):14-19

17 Han YP, Tuan TL, Wu H, Hughes M, Garner WL. TNF- $\alpha$ stimulates activation of pro-MMP2 in human skin through NF-(к) B mediated induction of MT1-MMP. J Cell Sci 2001;114(Pt 1): 131-139

18 Pires BR, Silva RCMC, Ferreira GM, Abdelhay E. NF-kappaB: two sides of the same coin. Genes (Basel) 2018;9(1):E24

19 Kim JH, Gupta SC, Park B, Yadav VR, Aggarwal BB. Turmeric (Curcuma longa) inhibits inflammatory nuclear factor (NF)-кB and NF-кB-regulated gene products and induces death receptors leading to suppressed proliferation, induced chemosensitization, and suppressed osteoclastogenesis. Mol Nutr Food Res 2012;56(3):454-465 